\title{
Coherence masking protection in speech sounds: The role of formant synchrony
}

\author{
PETER C. GORDON \\ University of North Carolina, Chapel Hill, North Carolina
}

\begin{abstract}
Three experiments were performed to examine listeners' thresholds for identifying stimuli whose spectra were modeled after the vowels $/ \mathrm{I} /$ and $/ \varepsilon /$, with the differences between these stimuli restricted to the frequency of the first formant. The stimuli were presented in a low-pass masking noise that spectrally overlapped the first formant but not the higher formants. Identification thresholds were lower when the higher formants were present than when they were not, even though the first formant contained the only distinctive information for stimulus identification. This indicates that listeners were more sensitive in identifying the first formant energy through its contribution to the vowel than as an independent percept; this effect is given the name coherence masking protection. The first experiment showed this effect for synthetic vowels in which the distinctive first formant was supported by a series of harmonics that progressed through the higher formants. In the second two experiments, the harmonics in the first formant region were removed, and the first formant was simulated by a narrow band of noise. This was done so that harmonic relations did not provide a basis for grouping the lower formant with the higher formants; coherence masking protection was still observed. However, when the temporal alignment of the onsets and offsets of the higher and lower formants was disrupted, the effect was eliminated, although the stimuli were still perceived as vowels. These results are interpreted as indicating that general principles of auditory grouping that can exploit regularities in temporal patterns cause acoustic energy belonging to a coherent speech sound to stand out in the auditory scene.
\end{abstract}

Contemporary research on the perception of complex sounds has yielded a number of phenomena that demonstrate interactions in the perceptual processing of acoustic energy at widely separated frequencies. These results have shown that traditional accounts of hearing, which emphasized independent sensory channels tuned to narrow frequency bands, must be augmented by across-frequency mechanisms that operate early in perceptual processing (Green, 1988; Hall, Haggard \& Fernandes, 1984; Yost, Sheft, \& Opie, 1989). At the same time as the latter developments in psychoacoustics have occurred, there has been substantial interest in "auditory scene analysis"-in how the auditory system parses acoustic stimulation into separate, coherent, auditory objects arising from events in the world (Bregman, 1990). The idea that basic auditory processes operating across frequencies play an important role in auditory scene analysis by helping to form coherent auditory objects has received considerable discussion (Yost \& Sheft, 1993). In the domain of speech per-

The research reported in this paper was supported by grants from the Stephenson Faculty Fund and by the University Research Council of the University of North Carolina at Chapel Hill. I would like to thank Søren Buus, Tom Carrell, John Grose, Joe Hall, and Bob Peters for helpful discussions of this research and comments on earlier versions of the paper. I would also like to thank Erika Manning for assistance in testing subjects. A preliminary report of these results was presented at the meetings of the Acoustical Society of America (June 1995). Direct correspondence to P. C. Gordon, Department of Psychology, University of North Carolina at Chapel Hill, Chapel Hill, NC 27599-3270 (e-mail: pcg@gibbs.oit.unc.edu). ception, the idea has received both support (Darwin, 1984a, 1984b) and criticism (Liberman \& Mattingly, 1989; Remez, Rubin, Berns, Pardo \& Lang, 1994). The goal of this paper is to examine further whether the coherence of speech sounds as auditory objects can be understood in terms of the auditory processes that underlie the perception of other complex sounds. This examination employs a task for examining whether acoustic energy is protected from masking if it is perceptually grouped into a coherent speech sound based on temporal variation in intensity at widely separated frequencies.

The phenomenon of comodulation masking release (CMR) provides clear evidence that auditory grouping can have a strong effect on the masked detection of simple signals (Hall \& Grose, 1990; Hall et al., 1994) and also of speech signals (Grose \& Hall, 1992). This phenomenon occurs when energy is added to a masker at frequencies that are distant from the signal. If the amplitude modulation of the added energy is unrelated to the masker, it has little effect on thresholds. If it has the same amplitude modulation as the masker, it causes a decrease in the threshold for detecting the signal. While a number of mechanisms potentially underlie this effect (Hall \& Grose, 1988), it is clear that grouping based on temporal patterns plays an important role (Hall \& Grose, 1990). The logic of CMR provides the basis for the task used here, except that instead of studying how coherence in the masker can release the signal from masking, we examine how coherence in the signal may provide protection from masking. This involves more than simply switching the terminology of 
what the masker is and what the signal is. In CMR studies, the elements of the masker that cohere have sufficient amplitude to be clearly audible, whereas the elements of the signal in the present task are barely audible. Further, the coherent maskers in CMR studies have relatively long durations, whereas the speech sounds studied in our experiments are brief. Finally, the present task examines target identification rather than target detection.

The experiments in this paper examine the identification of stimuli whose spectra are modeled after the vowels / $/ \mathbf{l}$ (as in "bit") and / $\varepsilon$ / (as in "bet"), a distinction that can be minimally cued by the frequency of the first formant. Since Darwin's initial work (Darwin, 1984a, 1984b), this distinction has been of great service in the study of the coherence of speech sounds, because the first formant is spectrally well separated from the higher formants and because of the acoustic simplicity of steady state vowels. For the present purposes, this allows the first formant to be masked with a stimulus that has little masking effect on the higher formants. Further, the temporal relations between the first formant and the higher formants can be manipulated easily.

\section{EXPERIMENT 1}

In Experiment 1, listeners' thresholds for identifying the vowels $/ \mathrm{I} /$ and $/ \varepsilon$ / were compared with their thresholds for identifying the distinctive portion of the vowels isolated from the remainder of the vowel by low-pass filtering. The targets were presented in noise that was low-pass filtered so that it spectrally overlapped the energy associated with the first formant but was far away from the second and third formants of the vowels. Comparing performance on full-vowel stimuli to first-formant-only stimuli allowed us to determine whether it was easier to identify the first formants on the basis of their their contribution to the vowel than to identify them in isolation.

\section{Method}

Subjects. Nine subjects participated in the study as part of a course requirement

Stimuli. Figure 1 illustrates the stimuli used in the experiment. Two vowel sounds (/c/ and /1/ ), modeled on stimuli used by Darwin and Gardner (1986), were created with a Klatt synthesizer. Both vowels shared the following characteristics: the fundamental frequency was $125 \mathrm{~Hz}$, the second formant had a center frequency of $2200 \mathrm{~Hz}$ with a bandwidth of $110 \mathrm{~Hz} 3 \mathrm{~dB}$ down from the peak amplitude, and the third formant had a center frequency of $2900 \mathrm{~Hz}$ with a bandwidth of $170 \mathrm{~Hz} 3 \mathrm{~dB}$ down from the peak amplitude. The two vowels differed in the following way: the center frequency of first formant for $/ \varepsilon /$ was $625 \mathrm{~Hz}$ (bandwidth of $50 \mathrm{~Hz} 3 \mathrm{~dB}$ down from the peak), whereas for $/ \mathrm{I} /$ it was $375 \mathrm{~Hz}$ (bandwidth of $50 \mathrm{~Hz} 3 \mathrm{~dB}$ down from the peak).

Both vowels were low-pass filtered beginning at $1000 \mathrm{~Hz}$ using a digital filter (Kaiser \& Reed, 1978) with a rolloff of $50 \mathrm{~dB}$ stopband attenuation and a $250-\mathrm{Hz}$ transition band. This was done to create sounds that lacked energy at the higher formants. (For con-

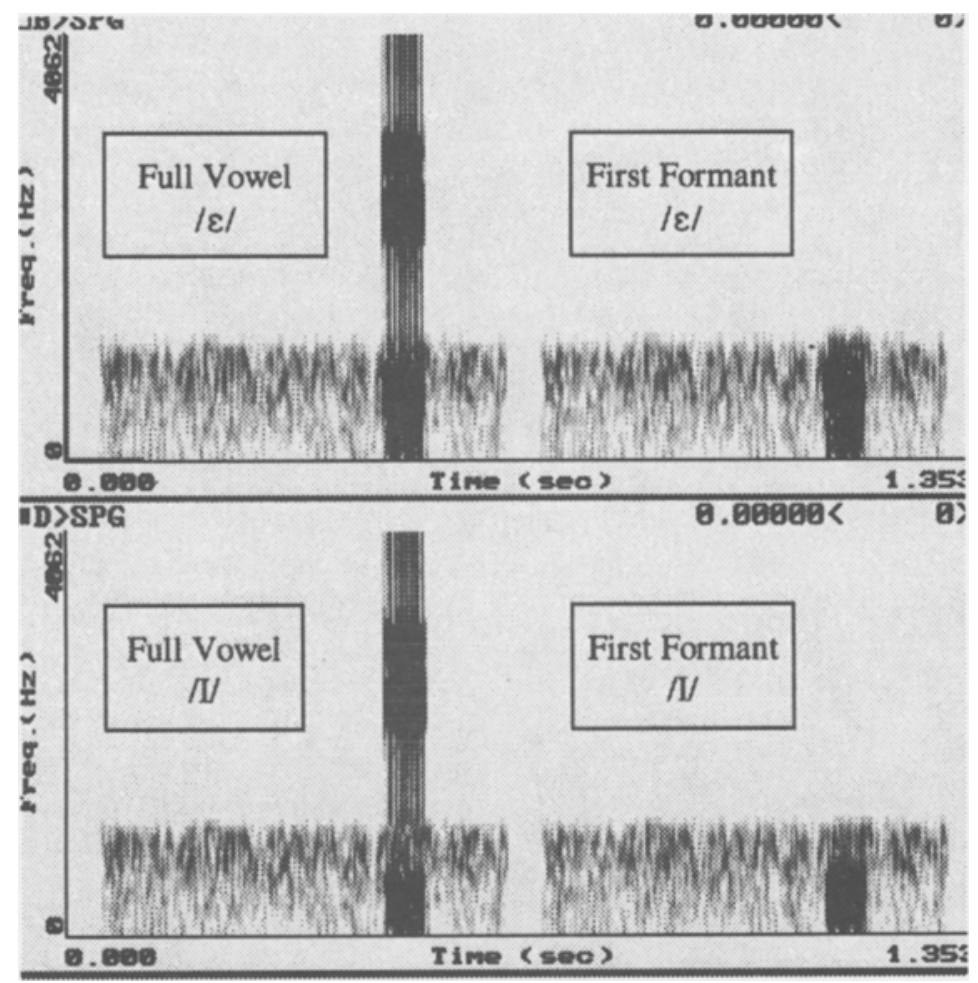

Figure 1. Spectrograms of the stimuli used in Experiment 1. The left column illustrates the stimuli in the full-vowel condition, and the right column illustrates the stimuli from the first-formant-only condition. 
venience, we refer to these as "first-formant-only" stimuli even though energy was present at the harmonics of the fundamental below $1000 \mathrm{~Hz}$ ). In addition, the vowel $/ \varepsilon /$ was high-pass filtered at $1250 \mathrm{~Hz}$ (rolloff of $50 \mathrm{~dB}$ and a pass-to-stop transition width of $250 \mathrm{~Hz}$ ) to create a sound that lacked the distinctive energy at the first formant. This high-pass portion of / $/$ / was digitally mixed with the two low-pass portions to create the "full-vowel" versions of $/ \varepsilon /$ and $/ \mathrm{I} /$ that were used in the study. This filtering and mixing was done, rather than directly using the output of the synthesizer, because in cascade synthesis the characteristics of a lower formant can influence the amount of energy present at higher formants. The filtering and mixing guaranteed that the full-vowel stimuli were identical above $1000 \mathrm{~Hz}$ and that the only information that could distinguish the two vowels was below $1000 \mathrm{~Hz}$. The amplitude of the low-pass portion (first-formant) was $12 \mathrm{~dB}$ higher than that of the high-pass portion (higher formants). The first-formant-only and full-vowel stimuli were shortened to $60 \mathrm{msec}$, which included 5-msec linear ramps at onset and offset, using a waveform editor.

The masker was a $600-\mathrm{msec}$ white noise, low-pass filtered at $1000 \mathrm{~Hz}$. It was presented at approximately $62 \mathrm{~dB}$ SPL. The target signal (first-formant only or full vowel) began $420 \mathrm{msec}$ after the onset of the masking noise.

Procedure and Design. On each trial, a single signal in noise was presented and subjects were asked to identify it by pressing the appropriate key. A one-up, three-down adaptive-tracking procedure was used to adjust the signal level in order to determine listeners' identification thresholds. Feedback was presented only for incorrect responses. The initial amplitude of the low-pass portion of the stimuli was $74 \mathrm{~dB}$ SPL. The step size of the signal adjustment was $8 \mathrm{~dB}$ for the first 2 reversals, $4 \mathrm{~dB}$ for the next 2 reversals, and $2 \mathrm{~dB}$ for the final 12 reversals in a run. In the full-vowel condition, the amplitude of both the low-pass and high-pass portions of the vowel were adjusted in the adaptive tracking. The average signal level of the last 8 reversals was taken as the threshold for the run.

Thresholds were measured in the following two conditions.

Identification of full-vowel stimuli $(/ \mathbf{\varepsilon} /$ or $/ \mathbf{I} /)$ : Listeners were told that they were to decide whether the signal sounded like the vowel / $\varepsilon$ / as in "bet" or / $\mathbf{l}$ as in "bit." They were to press the key labeled " 1 " if it was $/ \mathrm{I} /$ and " 2 " if it was $/ \boldsymbol{\varepsilon} /$.

Identification of first-formant-only stimuli: Listeners were told that they would hear either a somewhat low-pitched sound that might sound somewhat like a muffled version of $/ 1$ / or a higherpitched sound that might sound like a muffled version of $/ \varepsilon /$. They were to use the keys labeled " 1 " and " 2 " to identify these sounds. The listeners performed 10 runs in each condition, rotating through the conditions in the order listed above. The first two runs in each condition were considered practice and were not included in the analysis.

\section{Results}

Table 1 shows the mean signal level at threshold. Repeated measures analysis of variance showed that identification thresholds were lower in the full-vowel condition

Table 1

Results for Identification of Stimuli in Experiment 1

\begin{tabular}{|c|c|c|c|c|}
\hline \multirow[t]{3}{*}{$a$} & \multicolumn{4}{|c|}{ Condition } \\
\hline & \multicolumn{2}{|c|}{ Full Vowel } & \multicolumn{2}{|c|}{ First Formant Only } \\
\hline & $M$ & $S D$ & $M$ & $S D$ \\
\hline Signal level & 55.3 & 2.1 & 58.5 & 2.3 \\
\hline
\end{tabular}

Note - The mean signal levels (dB SPL) of the low-pass portions of the vowel at threshold for identification are shown as a function of the experimental conditions. Standard deviations are across subjects. than in the first-formant-only condition $[F(1,8)=23.4$, $p<.001]$.

\section{Discussion}

Identification thresholds were lower in the full-vowel condition than in the first-formant-only condition, even though the only acoustic basis for identifying the vowels was also present in the first-formant-only stimuli. This shows that the frequency of the first formant stands out against the masking noise to a greater degree when it is perceived as part of a vowel than when it is perceived on its own. We will call this effect coherence masking protection.

Coherence masking protection in speech sounds appears to be similar to a phenomenon reported by Whalen and Liberman (1987; but see Bailey \& Heriman, 1993). They examined an instance of duplex perception, in which acoustic energy simultaneously contributes to the perceptual identity of a speech sound and produces its own nonspeech percept (Liberman, Isenberg, \& Rackerd, 1981; Rand, 1974). Whalen and Liberman showed that duplex perception could be induced with binaural stimulation by varying the intensity of the third formant transition, a cue to the distinction between "da" and "ga" in their study. At high intensities, listeners heard both a speech sound and a chirp corresponding to the particular third formant transition. Systematic manipulation of the intensities showed that subjects had higher thresholds for identifying the chirps than for identifying the speech sounds. The present demonstration is stronger than that of Whalen and Liberman in two respects: (1) In their task, subjects received no feedback on accuracy, so the threshold difference may have resulted from the greater familiarity of the speech sounds compared to the chirps. In our task, subjects received both feedback and substantial practice. (2) In their task, acoustic energy in the "base" speech sound to which the chirp was added may have masked the emergence of the chirp as a distinct perceptual object while still allowing it to contribute to the auditory quality of the entire complex. In our task, thresholds for speech identification are compared with thresholds for identifying the distinctive acoustic energy alone separated from the speech base, thereby to avoid any intrastimulus masking.

\section{EXPERIMENT 2}

The vowel stimuli used in the first experiment contain two obvious acoustic bases for perceptual coherence, both of which have been carefully studied by Darwin and his colleagues. The first is that the acoustic energy across the spectrum is harmonically related to a common fundamental. The second is that the acoustic energy across the spectrum comes on and goes off at the same time. Thus, if coherence masking protection has its basis in general principles of auditory organization, that basis could be in the perception of pitch, temporal alignment, or both.

The effect of common pitch in the identification of vowels at suprathreshold levels depends somewhat on 
the task that listeners are given. Darwin (1981) found that exciting the formants of a vowel on different fundamentals had little effect on listeners' identifications when only a single vowel was presented, but that it had a clear effect when the stimulus could be perceived as one of two vowels. Subsequent research documented the effect of a common fundamental in the identification of vowel stimuli that were subject to alternative perceptual organizations because of the mistuning of a harmonic (Darwin \& Gardner, 1986), the addition of a tone at or near a harmonic (Roberts \& Moore, 1990), or the simultaneous presentation of two vowels (Scheffers, 1983, cited in Summerfield \& Assmann, 1989). Although the results of this research show that relation to a common fundamental has systematic effects on the identification of vowels and is a likely basis for their perceptual coherence, we will not review them carefully, because the present focus is on role of gross temporal structure in perceptual coherence; ongoing research in our lab addresses the role of a common fundamental in the effect observed in Experiment 1.

Research on the effects of gross temporal structure on the identification of vowels has a history similar to that on common pitch. Darwin (1981) found that starting and stopping formants at different times had little effect on identifications when a single vowel was present, but had a clear effect when the stimulus could support alternative perceptual organizations. In subsequent research, the effect of temporal structure on vowel identifications was explored by adding a harmonically related tone to a steady-state vowel sound in the vicinity of the first formant and systematically examining the effect of having the tone start before and/or end after the vowel (Darwin, 1984a, 1984b; Darwin \& Sutherland, 1984). The results show that the contribution of the tone to phonetic quality diminishes considerably when the tone begins $30 \mathrm{msec}$ before the vowel and continues to diminish as the lead increases. Vowels of relatively long duration (in the 300msec range) show contributions of the tone at longer leads than do relatively short vowels (in the $60-\mathrm{msec}$ range). Further, the contribution of a tone to vowel quality diminishes somewhat if it continues beyond the vowel; this effect of offset asynchrony is smaller than that of onset asynchrony, but it is consistently observed. The effect of offset asynchrony (Darwin \& Sutherland, 1984; Roberts \& Moore, 1991a), as well as the finding that the contribution of the tone to phonetic quality can be removed if it is perceptually captured by a surrounding sequence of tones (Darwin, Pattison, \& Gardner, 1989), indicates that these experiments assess auditory grouping, not just perceptual adaptation to the tone. Careful parametric experiments have shown that vowel identifications are more sensitive to leading tones than are pitch judgments, though it is not clear whether this is due to slower decision processes for pitch judgments than for phonetic identifications, or whether pitch perception and phonetic perception obey different principles of auditory grouping (Hukin \& Darwin, 1995).

In addition to the research on the effects of temporal alignment in the first formant region of vowels, research on duplex perception has provided evidence on the extent to which transitions of the third formant that precede a base speech stimulus contribute to the identification of consonants (Bentin \& Mann, 1990; Nygaard, 1993; Nygaard \& Eimas, 1990). Consistent with the results on vowels, these studies show that onset asynchronies in the range of $40 \mathrm{msec}$ are sufficient to substantially reduce the phonetic contribution of the leading stimulus, except in cases in which lexical factors promote integration ( $\mathrm{Ny}$ gaard, 1993).

In the present experiment (and the next), we examined whether coherence masking protection, as demonstrated in Experiment 1, would be influenced by the temporal structure of a vowel. This was done by determining identification thresholds for vowels in which the distinctive first formant was coterminous with the higher formants and for vowels in which the first formant and the higher formants were temporally misaligned. Performance on both of these types of stimuli was compared with performance on stimuli consisting of the distinctive first formant energy only. A fairly severe form of temporal misalignment was employed, which consisted of having the higher formants precede the first formant by $60 \mathrm{msec}$. Because the higher formants were only $60 \mathrm{msec}$ in duration, they never overlapped in time with the first formant. This severe form of misalignment was used in order to provide a strong experimental manipulation, and because initial stimulus construction made it clear that the tendency to perceptually integrate parts of the stimulus was promoted substantially by the presence of the masking noise. When heard at initial amplitude levels of signal and masker, these stimuli evoked in most listeners a duplex percept consisting of both a vowel and a tone whose pitch depended on the first formant. This effect of the masking noise on perceptual integration is consistent with findings by Hall and Peters (1981), who found that nonsimultaneous harmonics produced a virtual pitch in many listeners when presented in a moderate-level masking noise, but not when presented in silence. Because the present paradigm involved studying perceptual integration in noise, the use of this extreme form of temporal misalignment was possible.

One additonal change was made to the stimuli from the first experiment. Because the goal in the experiment was to examine the contribution of temporal alignment of formants to coherence masking protection, it was desirable to use stimuli in which the acoustic basis of perceiving a common pitch had been substantially minimized or eliminated. This was done by replacing the first formant (low-pass) components of the stimuli used in the first experiment with narrow bands of noise centered on the first formants, as is illustrated in Figure 2. Experiments by Roberts and Moore (1991b) have shown that aperidoic energy in the first formant region can influence the phonetic quality of vowels. Thus, in the present stimuli, the first formant was no longer supported by a harmonic series that progressed through the higher formants. Instead, the acoustic energy serving as the first formant was separated from the high-pass portion of the 


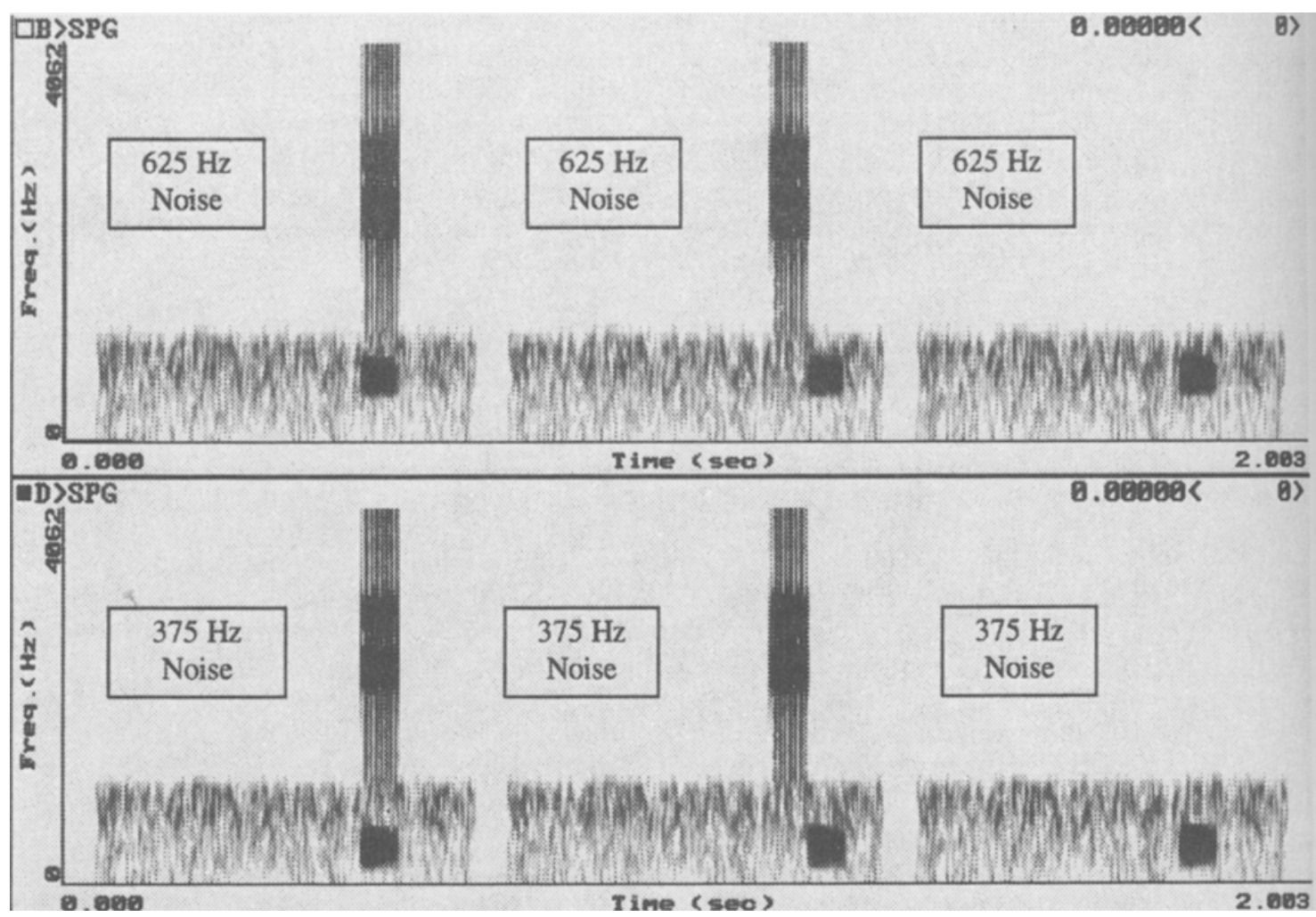

Figure 2. Spectrograms of the stimuli used in Experiment 2. The left column illustrates the synchronous-formants condition, the middle column illustrates the preceding-formants condition, and the right column illustrates the no-formants condition.

vowel by $600 \mathrm{~Hz}$ in the case of $/ \mathrm{I} /$ and $350 \mathrm{~Hz}$ in the case of $/ \varepsilon /$.

The use of the noise-band formants had an additional advantage besides eliminating harmonic relations as a potential source of auditory grouping. When the noise bands were presented alone in the masking noise without the higher formants, they sounded like tones. Further, the perceived pitch of the noises was very distinct, as one would expect given that the center frequency of one noise band was $375 \mathrm{~Hz}$ while for the other it was $625 \mathrm{~Hz}$. Accordingly, the instructions in the no-formants condition made no reference to vowel identity and instead asked listeners to identify the signal as a high-pitched or low-pitched tone, an identification that was very readily made. This provision of identification labels for the stimuli was more straightforward than it was for the analogous first-formant-only stimuli in the previous experiment. The more complex spectrum of those stimuli made them somewhat difficult to describe, and it could be argued that a weak relation between the stimulus and the identification label for those stimuli might be responsible for the higher thresholds observed for the first-formantonly stimuli as compared with the full-vowel stimuli. ${ }^{1}$ The very clear relation between stimulus and identifica- tion label for the noise-band stimuli undermines that argument as an account of relative performance in the present experiment.

\section{Method}

Subjects. Fifteen individuals participated in a single experimental session as part of a course requirement. None of them had participated in the previous experiment.

Stimuli, Procedure, and Design. Two $50-\mathrm{Hz}$ bands of noise, one centered on $375 \mathrm{~Hz}$ and the other on $625 \mathrm{~Hz}$, were used in place of the low-pass portions of the vowels $/ \varepsilon /$ or $/ \mathrm{I} /$ used in the previous experiment. The noisebands were made by passing a broadband $(0-2000 \mathrm{~Hz})$, constant spectrum level noise through a digital filter (IHR Universal) with extremely sharp spectral skirts and a noise floor over $70 \mathrm{~dB}$ down. The sampling rate of the filter was $2500 \mathrm{~Hz}$, and the output was low-pass filtered at 1250 and recorded onto digital audio tape. ${ }^{2}$ Playback of the tape was then redigitized at $10 \mathrm{kHz}$, using a Kay Elemetrics CSL system. The $50-\mathrm{Hz}$ bands of noise were edited into $60-\mathrm{msec}$ stimuli with 5 -msec onset and offset ramps. Nine different $60-\mathrm{msec}$ stimuli were made from each noise, so that the fluctuations present in the narrow bands of noise would not be the same in each stimulus presentation; the starting level of the signal was $69 \mathrm{~dB}$ SPL. The other stimulus features- the highpass portion of the vowel and the masking noise-were the same as in the previous experiment. In the synchronous-formants condition, both the noise band and the high-pass portion of the vowel began $420 \mathrm{msec}$ into the masker. In the preceding-formants con- 
Table 2

Results for Identification of Stimuli in Experiment 2

\begin{tabular}{|c|c|c|c|c|c|c|}
\hline & \multicolumn{6}{|c|}{ Condition } \\
\hline & \multicolumn{2}{|c|}{$\begin{array}{l}\text { Synchronous } \\
\text { Formants }\end{array}$} & \multicolumn{2}{|c|}{$\begin{array}{l}\text { Preceding } \\
\text { Formants }\end{array}$} & \multicolumn{2}{|c|}{ No Formants } \\
\hline & $M$ & $S D$ & $M$ & $S D$ & $M$ & $S D$ \\
\hline Signal level & 53.9 & 4.2 & 63.5 & 6.9 & 57.7 & 3.8 \\
\hline
\end{tabular}

Note-The mean signal levels (dB SPL) of the distinctive noise bands at threshold are shown as a function of the experimental conditions. Standard deviations are across subjects.

dition, the high-pass portion of the vowel began $360 \mathrm{msec}$ into the masker and the noise band began $60 \mathrm{msec}$ later. In the no-formants condition, the noise band began $420 \mathrm{msec}$ into the masker.

In the synchronous-formants condition, the subjects were given the same vowel-identification instructions as in the previous experiment. In the preceding-formants condition, the subjects were given the same vowel-identification instructions. They were also told that in addition to the vowel, they might hear a high- or a lowpitched tone on some of the trials. They were told to use whatever basis they thought would be effective for identifying the stimulus. In the no-formants condition, the subjects were told they would hear either a high-pitched tone or a low-pitched tone and were instructed as to the appropriate response keys to use for each. The subjects participated in $2 \mathrm{l}$ runs, rotating through the conditions in the order listed above. The first two runs in each condition were considered practice and were not included in the analysis.

\section{Results}

Table 2 shows the signal level at the identification threshold. Analysis of variance showed that performance in the three conditions differed significantly $[F(2,28)=$ $21.0, p<.001]$. One contrast showed that identification thresholds were lower in the synchronous-formants condition than in the no-formants condition $[F(1,14)=7.9$, $p<.025]$. A second contrast showed that thresholds were significantly lower in the no-formants condition than in the preceding-formants condition $[F(1,14)=18.6, p<$ $.005]$. Thresholds were also significantly lower in the synchronous-formants condition than in the precedingformants condition $[F(1,14)=31.8, p<.001]$.

As is shown in Table 2 , the variability across listeners was considerably larger in the preceding-formants condition than in the other conditions. This indicates that mean thresholds do not tell the complete story. Accordingly, the subjects were divided into two groups by using a median split on performance in the preceding-formants condition; the results are shown in Table 3. The breakdown

Table 3

Results for Identification of Stimuli in Experiment 2

\begin{tabular}{lccc}
\hline & \multicolumn{3}{c}{ Condition } \\
\cline { 2 - 3 } Median Split & $\begin{array}{c}\text { Synchronous } \\
\text { Formants }\end{array}$ & $\begin{array}{c}\text { Preceding } \\
\text { Formants }\end{array}$ & No Formants \\
\hline Signal level & & & \\
Top half & 52.6 & 57.3 & 56.5 \\
Bottom half & 55.0 & 68.9 & 58.8 \\
\hline
\end{tabular}

Note-The mean signal levels ( $\mathrm{dB}$ SPL) of the distinctive noise bands at threshold are shown as a function of the experimental conditions and broken down by a median split on performance in the noise-bandwith-preceding-formants condition. shows two things: First, performance in the synchronousformants condition is better than that in the other two conditions, regardless of the breakdown. Second, some subjects had a great deal of difficulty with the precedingformants condition, whereas others did not. However, even the subjects who were selected on the basis of their good performance in the preceding-formants condition did no better in that condition than in the no-formants condition.

\section{Discussion}

The results of the experiment provide a second demonstration of coherence masking protection. Identification thresholds were lower in the synchronous-formants condition than in the no-formants condition. The magnitude of this difference $(3.8 \mathrm{~dB})$ obtained with a noiseband first formant is comparable to that observed with a harmonically supported first formant in Experiment 1 (3.2 dB). This result shows that coherence masking protection is observed in comparisons of identification thresholds between vowels and noise bands that have very clear identification labels as low- and high-pitched tones. It also shows that a common pitch is not necessary to obtain coherence masking protection.

The results of the experiment show that there was no coherence masking protection for stimuli in which the higher formants were temporally misaligned with the lower formants, even though the misaligned stimuli were sufficient to produce a phonetic percept. This finding is consistent with the idea, supported by findings on suprathreshold identifications, that temporal alignment plays a role in auditory grouping. However, in this experiment a fairly severe manipulation of temporal alignment (nonsimultaneity) was used in order to disrupt temporal structure. Accordingly, in the next experiment, a temporal manipulation was used that was more in line with those employed in previous research (Darwin, 1984a, 1984b).

\section{EXPERIMENT 3}

The goal in this experiment was to provide a stronger test of the finding from the previous experiment that temporal alignment of formants within a vowel is a necessary condition for coherence masking protection (at least in the absence of a harmonic relation between the first formant and the higher formants). In the previous experiment, the temporal relation between the formants was severely disrupted by having the higher formants begin and end before the first formant began. In the present experiment, the first formant and higher formants did overlap in time, but did not have common onsets or offsets. Instead, the higher formants started $40 \mathrm{msec}$ before the first formant did, and ended $40 \mathrm{msec}$ after it ended. Previous research (Darwin, 1984a, 1984b; Darwin \& Sutherland, 1984) has shown that leads and lags of $40 \mathrm{msec}$ can have considerable influence on what information is integrated into a phonetic judgment. In this experiment, we compared identification thresholds for noise bands with synchronous higher formants, noise 
bands with "fringing" higher formants that led and lagged by $40 \mathrm{msec}$, and noise bands with no formants (see Figure 3). These comparisons allowed us to determine whether coherence masking protection would depend on this level of temporal alignment within a signal.

\section{Method}

Subjects. Nine individuals participated in a single session and were paid at a rate of $\$ 6 / \mathrm{h}$. They were recruited with posted notices. None had participated in the earlier experiments.

Stimuli, Procedure, and Design. The distinctive noise bands were the same as in the previous experiment, except that they were shortened to $40 \mathrm{msec}$ with $5-\mathrm{msec}$ onset and offset ramps. In the synchronous-formants condition, both the noise band and the highpass portion of the vowel began $420 \mathrm{msec}$ into the masker and ended $40 \mathrm{msec}$ later. In the fringing-formants condition, the highpass portion of the vowel began $380 \mathrm{msec}$ into the masker and ended $120 \mathrm{msec}$ later; the 40 - $\mathrm{msec}$ noise band began $420 \mathrm{msec}$ into the masker. In the no-formants condition, the $40-\mathrm{msec}$ noise band began $420 \mathrm{msec}$ into the masker.

In the synchronous-formants condition, the subjects were given the same vowel-identification instructions as in the previous experiment. In the fringing-formants condition, the subjects were given the same vowel-identification instructions but were told that the vowels would be of longer duration. In the no-formants condition, the subjects were told that they would hear either a high-pitched tone or a low-pitched tone and were instructed as to the appropriate response keys to use for each.
All other aspects of the procedure were the same as those for the previous experiment.

\section{Results}

Table 4 shows the mean signal level at threshold. Analysis of variance showed that performance in the three conditions differed significantly $[F(2,16)=8.8$, $p<.005]$. Contrasts showed that thresholds were lower in the synchronous-formants condition than in the noformants condition $[F(1,8)=20.8, p<.005]$ and the fringing formants condition $[F(1,8)=9.2, p<.025]$. The identification thresholds did not differ significantly between the fringing-formants and the no-formants conditions $[F(1,8)=2.2, p>.15]$.

\section{Discussion}

Consistent with both of the previous experiments, the results show coherence masking protection; thresholds were lower for the noise bands with synchronous higher formants than they were for noise bands with no formants. Consistent with Experiment 2, the results show that the temporal relation between the higher formants and the masked lower formant is a critical determinant of coherence masking protection. When the higher formants began before and ended after the noise band, no coherence

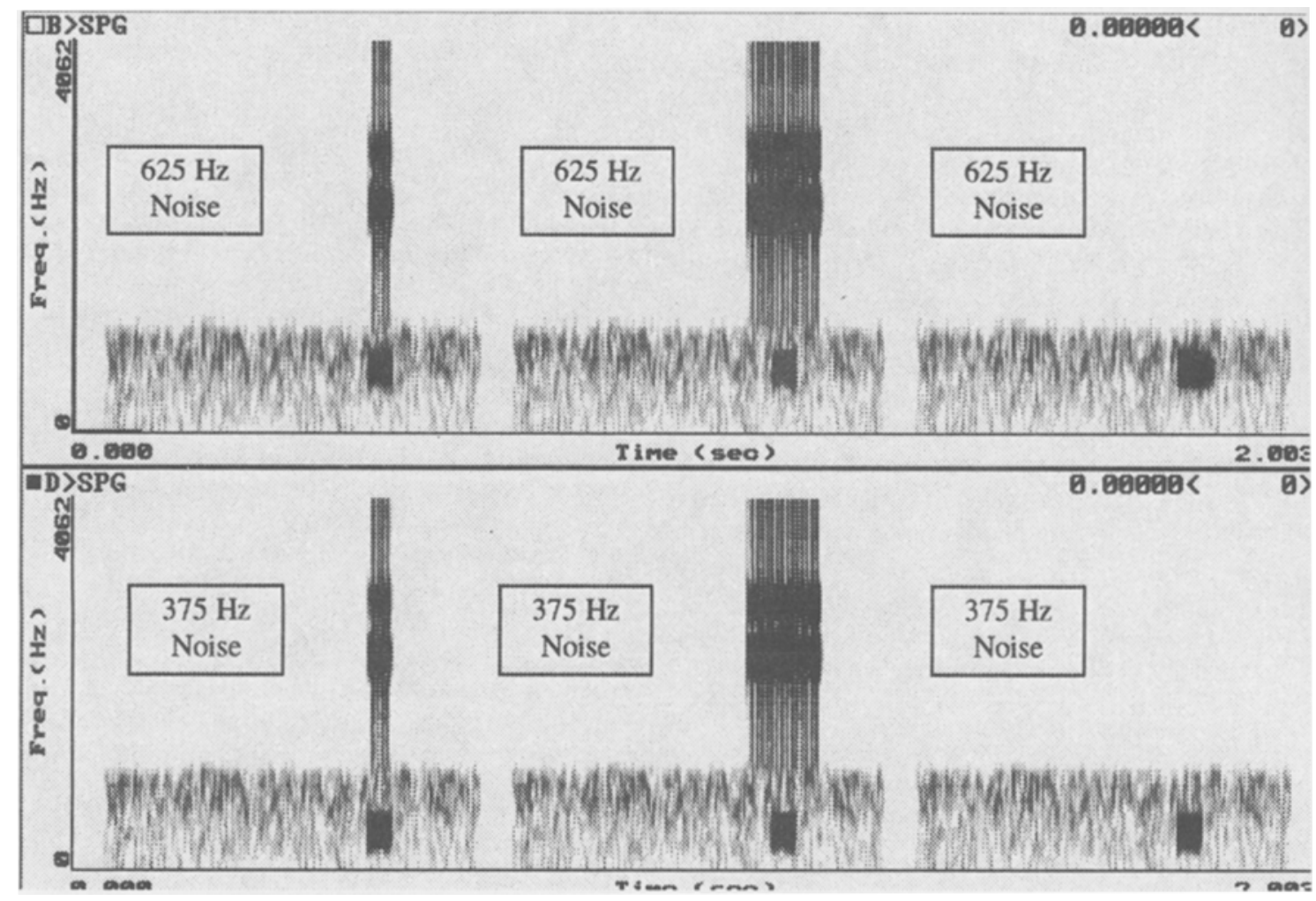

Figure 3. Spectrograms of the stimuli used in Experiment 3. The left column illustrates the synchronous-formants condition, the middle column illustrates the fringing-formants condition, and the right column illustrates the no-formants condition. 
Table 4

Results for Identification of Stimuli in Experiment 3

\begin{tabular}{|c|c|c|c|c|c|c|}
\hline & \multicolumn{6}{|c|}{ Condition } \\
\hline & \multicolumn{2}{|c|}{$\begin{array}{l}\text { Synchronous } \\
\text { Formants }\end{array}$} & \multicolumn{2}{|c|}{$\begin{array}{l}\text { Fringing } \\
\text { Formants }\end{array}$} & \multicolumn{2}{|c|}{ No Formants } \\
\hline & $M$ & $S D$ & $M$ & $S D$ & $M$ & $S D$ \\
\hline Signal level & 57.5 & 4.1 & 63.3 & 5.1 & 61.5 & 3.6 \\
\hline
\end{tabular}

Note-The mean signal levels (dB SPL) of the distinctive noise bands at threshold are shown as a function of the experimental conditions. Standard deviations are across subjects.

masking protection was observed, even though the two components of the sound were simultaneously present and were sufficient to generate consistent phonetic percepts. The finding that coherence masking protection is eliminated by a $40-\mathrm{msec}$ misalignment of the onsets and offsets of the first formant relative to the higher formants is consistent with previous work (Darwin, 1984a, 1984b; Darwin \& Sutherland, 1984) on how common onsets and offsets affect auditory grouping in vowel identification. The previous studies looked at the contribution to vowel identification of an added tone when all signals are presented at high amplitudes in a quiet background. The present study looks at a very different measure of coherence, protection from masking, in sounds presented at low signal-to-noise ratios. The results of the present study are also consistent with those of Darwin (1981), which showed that large asynchronies in the onsets of formants did not affect vowel identification when the acoustic information supported the perception of only a single vowel and when the stimuli were presented at high signal-tonoise ratios. In the present study, the vowel stimuli with formant asynchronies were readily identified at high signal-to-noise ratios. However, synchrony between formants was shown to play an important role in coherence when the task was made difficult by low signal-to-noise ratios, a condition not examined by Darwin (1981).

\section{GENERAL DISCUSSION}

The three experiments in this paper demonstrate coherence masking protection, a phenomenon in which identification thresholds for acoustic energy are lower when they are based on that energy's distinctive contribution to the identity of a coherent complex sound, such as a phonetic segment, than when the energy must be identified on its own. It occurs when the distinctive acoustic energy is spectrally well separated from the other acoustic energy that contributes to the perceived sound. Further, it is strongly influenced by the gross temporal structure of the stimulus. Below, we address the significance of this phenomenon for an understanding of levels at which perceptual coherence occurs and also discuss mechanisms that may underlie it.

\section{Types of Perceptual Coherence}

Measures of perceptual coherence. Perceptual coherence has been studied mostly by examining whether some simple component contributes to the identification of a complex stimulus. This can be called the "part-to-whole" method. It has been used in studies of a great range of topics, ranging from the psychoacoustic modeling of the pitch and phonetic quality of short sounds (Darwin, 1984a; Darwin \& Gardner, 1986; Moore, Glasberg, \& Peters, 1985), to the duplex perception of consonants (Rand, 1974; Liberman et al., 1981), to the identification of replicas of sentences composed of time-varying sinusoids (Remez, Rubin, Pisoni \& Carrell, 1981). Although this method is undoubtedly useful, it has the limitation of equating coherence with identification. Human pattern recognition is flexible and open to strategies, particularly when the stimulus is impoverished and the called-for identification is at an abstract level. It is likely that we can identify objects in cases where they do not have a high level of subjective coherence. This has led to debates over whether specific research findings reflect true perceptual effects or higher level strategic effects (e.g., Nusbaum, 1984; Nusbaum, Schwab, \& Sawusch, 1983; Repp, 1984; Repp, Milburn, \& Ashkenas, 1983). Thus, in some cases the finding that a stimulus component contributes to the identification of a complex stimulus may reflect optional, higher level, strategic processes rather than early processes that establish primary perceptual coherence.

An alternative method for studying coherence is to examine whether being part of a complex stimulus influences what can be done perceptually with a component of the stimulus, as was done in Experiments 1-3. This can be called the "whole-on-part" method, and it has also been used in a range of previous studies. Research on streaming has shown that the order of component tones within an auditory stream can be easily perceived, but that it is very difficult to perceive the order between streams (Bregman \& Campbell, 1971). Research on comodulation masking release shows that energy in a frequency band produces less masking of a target signal within that band when its envelope matches that of other masking bands than when it does not (Hall et al., 1984). These diverse whole-on-part procedures share the feature that acoustic factors determine the possible perceptual organization of the stimulus, which in turn has a powerful influence on listeners' perception of parts of the stimulus. These types of perceptual organization appear to be less open to flexible perceptual strategies, because if they were open to strategies, listeners would reorganize the percepts to be optimal for the task that the listeners must perform on parts of the stimulus.

Of course, there is no necessity that the results of partto-whole and whole-on-part methods diverge. The dependence of coherence masking protection on the simultaneous onset and offset of formants is consistent with the results of studies examining whether added tones influence vowel identification (Darwin, 1984a, 1984b). The consistency of the results with these two different methods suggests that the same basic processes of auditory grouping operate in identifying vowels presented at both high and low signal-to-noise ratios.

Levels of perceptual coherence. The possible levels at which perceptual coherence occurs have been the sub- 
ject of much discussion; for example, Cutting (1976) concluded that fusion of dichotically presented speech stimuli occurs at three or possibly four levels of perceptual analysis. However, the question of whether the perception of speech involves any of the grouping processes of general auditory perception has continued to be a matter of considerable debate.

Liberman and his colleagues (Liberman \& Mattingly, 1989; Whalen \& Liberman, 1987) have advanced the argument that phonetic perception depends entirely on its own processes of grouping, which are independent of those of general auditory grouping. The phenomenon of duplex perception (Liberman et al., 1981; Rand, 1974)that acoustic energy can be perceived as speech and as nonspeech simultaneously - has been used to support this argument. In this regard, Whalen and Liberman (1987) have provided a particularly dramatic result by showing that the threshold for identifying a nonspeech chirp is higher than the threshold for identifying the consonant to which it provides the only distinctive information. They argue that this shows that there are distinct phonetic and auditory ways of perceiving sounds, and that phonetic perception does not build on auditory perception, because it can use acoustic information that is not available to the auditory system. Whalen and Liberman's argument is compelling, but not conclusive. The integration of acoustic energy into the phonetic percept may have involved general auditory principles of perceptual integration even though the task called for a speech identification. Indeed, Ciocca and Bregman (1989) found that a chirp of the sort studied by Whalen and Liberman could be captured perceptually by a train of chirps and that this reduced its contribution to phonetic identification. Such a finding is consistent with a two-stage model of speech perception in which an initial grouping of acoustic energy provided by general auditory mechanisms is followed by application of recognition schemas that are specific to the structure of speech (Bregman, 1990). Remez et al. (1994) have countered that the two-stage model is virtually untestable, because it can, after the fact, attribute the effect of any acoustic factor to the general auditory stage of processing or to the speech-specific stage of processing.

The phenomenon reported here, coherence masking protection, could be due either to speech-specific mechanisms or to general auditory processes. The results of Experiments 2 and 3 indicate that the mechanism of coherence makes use of gross temporal alignment across the spectrum. Since such alignment results from the way in which speech sounds are produced, there would be adaptive value in having speech-specific perceptual mechanisms tuned to this sort of temporal structure. However, many nonspeech events produce sounds with gross temporal alignment across the spectrum, providing adaptive value to general mechanisms for exploiting this facet of temporal structure. Temporal alignment similar to that studied here has clear effects on the identification of speech and nonspeech sounds, a pattern that is parsimo- niously explained by appeal to general principles of auditory grouping (Darwin, 1984a). Such an explanation of coherence masking protection in terms of general auditory mechanisms would be justified if the effect were shown to occur for nonspeech stimuli under the conditions studied here. Alternatively, if coherence masking protection were not observed in nonspeech stimuli, an appeal to speech-specific mechanisms would be justified.

Gordon (1996) has shown that nonspeech noise complexes, whose temporal characteristics match those of the vowels in Experiment 3, show the same pattern of coherence masking protection as do the vowels. The finding of similar effects with speech sounds and complex nonspeech sounds is parsimoniously explained by the operation of general principles of auditory grouping. That finding, along with the present results, indicates that general auditory mechanisms give speech sounds an important level of perceptual coherence when that is possible. When that is not possible, speech-specific recognition schemas may still allow identification of a speech sound (as in the case of the temporally disrupted vowels of Experiments 2 and 3), but these sounds lack the kind of perceptual coherence that helps them stand out in the auditory scene. Thus, the initial stage of the two-stage speech recognition model plays a very important, though not completely necessary, role.

\section{Possible Mechanisms of Coherence Masking Protection}

Coherence masking protection involves a reduction in the threshold for identifying acoustic energy on the basis of its contribution to the perception of a complex auditory object (a vowel) as compared with the threshold for identifying that same energy in isolation. The effect can be obtained with signals in which the acoustic energy is spectrally far apart, and it is influenced by the gross temporal structure of the stimulus. As such, it is similar to CMR (Hall et al., 1984) in that it involves a reduction in masking produced by envelope patterns across frequencies. Accordingly, the literature on CMR offers a source of possible explanations of coherence masking protection.

One explanation of CMR (Buus, 1985) is that the comodulated flanking bands facilitate perception in minima of the on-signal masking band ("listening in the valleys," or dip listening). This mechanism appears to account for CMR effects in some circumstances, though not in others (Grose \& Hall, 1989; Hall \& Grose, 1988). As it is conceived for CMR, dip listening does not account for coherence masking protection, because the higher formants of the vowel are unrelated to fluctuations in the masker. However, the basic notion of facilitating location of intervals with favorable signal-to-masker ratios could be reformulated as "peak listening," in which the amplitude maximum in the higher formants provides information about the interval containing the amplitude maximum in the distinctive first formant. This mechanism accounts for the presence of coherence masking protection in the present experiments, but does not easily ac- 
count for the absence of coherence masking protection when the higher formants lead and lag the first formant in Experiment 3; in those stimuli, the peak in the first formant occurs during the peak of the higher formants. To account for the latter finding, peak listening could be modified so that the signal-to-masker ratio in the channels tuned to the possible distinctive signals would be averaged over an interval that depended on the time that the higher formants were at their maximum amplitude. On such an account, the leading and lagging higher formants of Experiment 3 would result in worse performance than would the synchronous higher formants, since the averaging would include intervals during which the distinctive signal was not present. In this way, the modified peak-listening model could account for the present results, and it therefore merits further consideration.

A second explanation of CMR is based on the ability of the auditory system to detect correlations in envelope fluctuations at different frequency bands (Richards, 1987); detectability of the signal is enhanced by comodulation, because the signal disrupts the correlation between masking bands. In the present experiments, coherence masking protection was observed only when the envelopes of the first and higher formants were the same (and thus perfectly correlated). Because envelope correlation promotes auditory grouping (Hall \& Grose, 1990), this would lead to perception of the formants as a single auditory complex. Theoretical treatments of profile analysis (Green, 1988), then, suggest a way in which the first formant could make a greater contribution to the identification of the resulting auditory complex than it could to its own independent identification. The essential idea is that the higher formants provide a concurrent perceptual basis for estimating the expected energy level at the frequency of the distinctive formant, which can be compared with the perceived level at that frequency. This comparison is more sensitive than the within-channel comparison of energy level in the first formant region when the signal is on and when it is off.

The results presented here do not provide a basis for determining whether either of these models is correct. The results do show a strong connection between phenomena of speech perception and psychoacoustics, and they point the way toward a deeper understanding of the role of general auditory processes in the perception of speech.

\section{REFERENCES}

Bailey, P. J., \& Herrman, P. (1993). A reexamination of duplex perception evoked by intensity differences. Perception \& Psychophysics, 54, 20-32.

BENTIN, S., \& MANN, V. A. (1990). Masking and stimulus intensity effects on duplex perception: A confirmation of the dissociation between speech and nonspeech modes. Journal of the Acoustical Society of America, 88, 64-74.

Bregman, A. S. (1990). Auditory scene analysis. Cambridge, MA: MIT Press.

Bregman, A. S., \& CAMPBell, J. (1971). Primary auditory stream segregation and the perception of order in rapid sequences of tones. Journal of Experimental Psychology, 89, 244-249.
BuUs, S. (1985). Release from masking caused by envelope fluctuations. Journal of the Acoustical Society of America, 78, 1958-1965. Ciocca, V., \& Bregman, A. S. (1989). The effects of auditory streaming on duplex perception. Perception \& Psychophysics, 46, 39-48. CutTing, J. E. (1976). Auditory and linguistic processes in speech perception: Inferences from six fusions in dichotic listening. Psychological Review, 83, 114-140.

DARWIN, C. J. (1981). Perceptual grouping of speech components differing in fundamental frequency and onset-time. Quarterly Journal of Experimental Psychology, 33A, 185-207.

DARWIN, C. J. (1984a). Auditory processing and speech perception. In H. Bouma \& D. G. Bouwhuis (Eds.), Attention and performance X: Control of language processes (pp. 197-210). Hillsdale, NJ: Erlbaum.

DARWIN, C. J. (1984b). Perceiving vowels in the presence of another sound: Constraints on formant perception. Journal of the Acoustical Society of America, 76, 1636-1647.

DARWIN, C. J., \& GARDNER, R. B. (1986). Mistuning a harmonic of a vowel: Grouping and phase effects on vowel quality. Journal of the Acoustical Society of America, 79, 838-845.

Darwin, C. J., PAtTison, H., \& Gardner, R. B. (1989). Vowel quality changes produced by surrounding tone sequences. Perception $\&$ Psychophysics, 45, 333-342.

Darwin, C. J., \& Sutherland, N. S. (1984). Grouping frequency components of vowels: When is a harmonic not a harmonic? Quarterly Journal of Experimental Psychology, 36A, 193-208.

GORDON, P. C. (1996). Coherence masking protection in brief noise complexes: Effects of temporal alignment. Manuscript submitted for publication.

Green, D. M. (1988). Profile analysis: Auditory intensity discrimination. New York: Oxford University Press.

Grose, J. H., \& HALL, J. W., III (1989). Comodulation masking release using SAM tonal complex maskers: Effects of modulation depth and signal position. Journal of the Acoustical Society of America, 85, 1276-1284.

Grose, J. H., \& HALL, J. W., III ( 1992). Comodulation masking release for speech stimuli. Journal of the Acoustical Society of America, 91, 1042-1050.

Hall, J. W., III, \& Grose, J. H. (1988). Comodulation masking release: Evidence for multiple cues. Journal of the Acoustical Society of America, 84, 1669-1675.

HALl, J. W., III, \& GRose, J. H. (1990). Comodulation masking release and auditory grouping. Journal of the Acoustical Society of America, 88, 119-125.

Hall, J. W., III, Haggard, M. P., \& Fernandes, M. A. (1984). Detection in noise by spectro-temporal pattern analysis. Journal of the Acoustical Society of America, 76, 50-56.

Hall, J. W., III, \& Peters, R. W. (1981). Pitch for nonsimultaneous harmonics in quiet and noise. Journal of the Acoustical Society of America, 69, 509-513.

Hukin, R. W., \& Darwin, C. J. (1995). Comparison of the effect of onset asynchrony on auditory grouping in pitch matching and vowel identification. Perception \& Psychophysics, 57, 191-196.

KAISER, J. F., \& REED, W. A. (1978). Bandpass (bandstop) digital filter design routine. Review of Scientific Instrumentation, 48, 1103-1106.

Liberman, A. M., IsEnberG, D., \& Rackerd, B. (1981). Duplex perception for stop consonants: Evidence for a phonetic mode. Perception \& Psychophysics, 30, 133-143.

Liberman, A. M., \& Mattingly, I. G. (1989). A specialization for speech perception. Science, 243, 489-494.

Moore, B. C. J., Glasberg, B. R., \& Peters, R. W. (1985). Relative dominance of individual partials in determining the pitch of complex tones. Journal of the Acoustical Society of America, 77, 18531860.

Nusbaum, H. C. (1984). Possible mechanisms of duplex perception: "Chirp" identification versus dichotic fusion. Perception \& Psychophysics, 35, 94-101.

Nusbaum, H. C., Schwab, E. C., Sawusch, J. R. (1983). The role of "chirp" identification in duplex perception. Perception \& Psychophysics, 33, 323-332.

NygaARD, L. C. (1993). Phonetic coherence in duplex perception: Ef- 
fects of acoustic differences and lexical status. Journal of Experimental Psychology: Human Perception \& Performance, 19, 268-286.

NyGAaRD, L. C., \& Eimas, P. D. (1990). A new version of duplex perception: Evidence for phonetic and nonphonetic fusion. Journal of the Acoustical Society of America, 88, 75-86.

RAND, T. C. (1974). Dichotic release from masking for speech. Journal of the Acoustical Society of America, 55, 678-680.

Remez, R. E., Rubin, P. E., Berns, S. M., Pardo, J. S., \& Lang, J. M. (1994). On the perceptual organization of speech. Psychological Review, 101, 129-156.

Remez, R. E., Rubin, P. E., Pisoni, D. B., \& Carrell, T. D. (1981). Speech perception without traditional speech cues. Science, 212, 947-950.

REPP, B. H. (1984). Against a role of "chirp" identification in duplex perception. Perception \& Psychophysics, 35, 89-93.

Repp, B. H., Milburn, C., \& Ashienas, J. (1983). Duplex perception: Confirmation of fusion. Perception \& Psychophysics, 33, 333-337.

RICHARDS, V. M. (1987), Monaural envelope correlation perception. Journal of the Acoustical Society of America, 82, 1621-1630.

ROBERTS, B., \& MOORE, B. C. J. (1990). The influence of extraneous sounds on the perceptual estimation of first-formant frequency in vowels. Journal of the Acoustical Society of America, 88, 25712583.

RoBerts, B., \& MOORE, B. C. J. (1991a). The influence of extraneous sounds on the perceptual estimation of first-formant frequency in vowels under conditions of asynchrony. Journal of the Acoustical Society of America, 89, 2922-2932.

Roberts, B., \& MOORE, B. C. J. (1991b). Modeling the effects of ex- traneous sounds on the perceptual estimation of first-formant frequency in vowels. Journal of the Acoustical Society of America, 89, 2933-2951.

SCHEFFERS, M. T. M. (1983). Sifting vowels: Auditory pitch analysis and sound segregation. Unpublished doctoral dissertation, University of Groningen.

Summerfield, Q., \& Assmann, P. F. (1989). Auditory enhancement and the perception of concurrent vowels. Perception \& Psychophysics, 45, 529-536.

Whalen, D. H., \& Liberman, A. M. (1987). Speech perception takes precedence over nonspeech perception. Science, 237, 169-171.

Yost, W. A., \& SheFT, S. (1993). Auditory perception. In W. A. Yost, A. N. Popper, \& R. R. Fay (Eds.), Human psychophysics (pp. 193236). New York: Springer-Verlag.

Yost, W. A., Sheft, S., \& OpIE, J. (1989). Modulation interference in detection and discrimination of amplitude modulation. Journal of the Acoustical Society of America, 86, 2138-2147.

\section{NOTES}

1. That argument can be countered directly by noting that subjects received immediate accuracy feedback throughout their extensive work with those stimuli.

2. My thanks to John Grose for making the noise bands.

(Manuscript received September 29, 1995; revision accepted for publication April 5, 1996.) 